\title{
The Viscous Properties of Diols \\ I. The Homologous Series of 1,2- and 1,n-Alkanediols
}

Grzegorz Czechowski and Jan Jadżyn

Institute of Molecular Physics, Polish Academy of Sciences,

M. Smoluchowskiego 17, 60-179 Poznań, Poland

Reprint requests to Prof. J. J.; Tel. Fax: +4861 8684-524; E-mail: jadzyn@ifmpan.poznan.pl

Z. Naturforsch. 58a, 317 -320 (2003); received January 18, 2003

The paper presents the results of viscosity measurements performed on 1,2-alkanediols, $\mathrm{H}\left(\mathrm{CH}_{2}\right)_{n-2} \mathrm{CH}(\mathrm{OH}) \mathrm{CH}_{2}(\mathrm{OH}), n=2: 12$, and $1, n$-alkanediols, $\mathrm{HO}\left(\mathrm{CH}_{2}\right)_{n} \mathrm{OH}, n=2: 10$, as functions of the temperature. It is shown that the viscosity (at constant temperature) and activation energy of the viscosity show a quite different dependence on the length of the alkane chain in 1,2- and 1,nalkanediols molecules.

Key words: Shear Viscosity; Alkanediols; Hydrogen Bonds; Self-association.

\section{Introduction}

The momentum transport in fluids subjected a velocity gradient can be described by the shear viscosity, defined by the shear stress divided by the velocity gradient. Numerous papers on viscosity simulations have been published $[1-10]$.

The paper presents the results of viscosity measurements on two series of diols: 1,2 - and 1,n-alkanediols. The intermolecular hydrogen bonds $\mathrm{O}-\mathrm{H}$...O make the compounds highly self-associated, and for not too long alkane chains $(n<6)$ the temperature range of the liquid phase is exceptionally large.

\section{Experiment}

The shear viscosity was measured with a Haake viscometer RV20 and the measuring system CV100. It consists of a rotary beaker filled with the studied liquid and a cylinder sensor of the Mooney-Ewart type (ME15), placed in the center of the beaker. The liquid gap was $0.5 \mathrm{~mm}$. The studied liquids show Newtonian behavior in the available range of shear rates $\left(30 \mathrm{~s}^{-1}\right.$ $-300 \mathrm{~s}^{-1}$ ). The accuracy of the viscosity determinations was $0.5 \%$. The temperature of the sample was controlled within $\pm 0.1^{\circ} \mathrm{C}$.

The purity and the viscosities of the diols are given in $[11,12]$.

\section{Results and Discussion}

Figures 1 and 2 present the temperature dependences of the viscosities of two series of diols, differ-

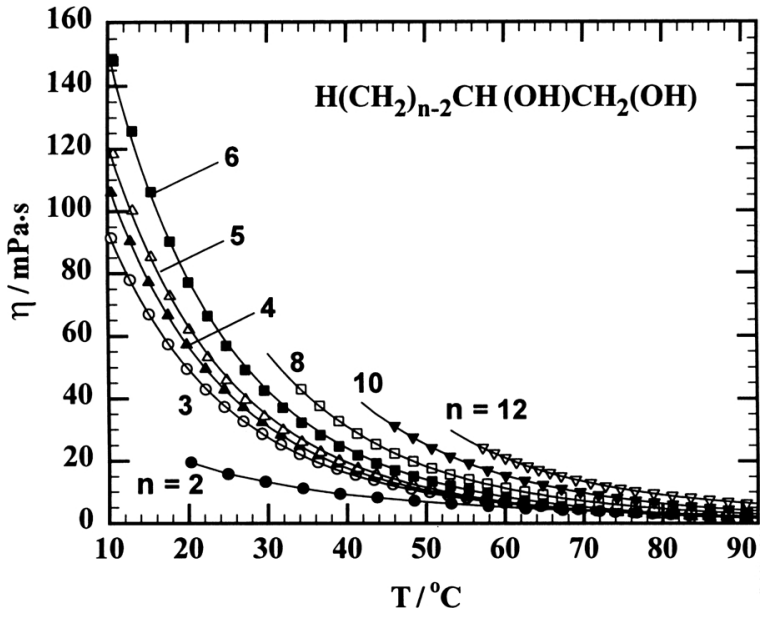

Fig. 1. Temperature dependences of the viscosity of 1,2-alkanediols. The solid lines represent the best fit of (3) to the experimental viscosity data.

ing in the location of the two hydroxyl groups. The location determines the intermolecular structures due to the hydrogen bonds between $\mathrm{O}-\mathrm{H}$ groups. Two hydroxylic groups joined with two neighboring carbon atoms at the end of the hydrocarbon chain make a distinct separation of the hydrophobic and hydrophilic parts of 1,2-alkanediol molecules. In such a situation, the hydrogen bonds $\mathrm{O}-\mathrm{H}$...O lead to rather cyclic, micelle-like structures. On the other hand, two terminal $\mathrm{O}-\mathrm{H}$ groups, separated by the hydrocarbon chain, in $1, n$-alkanediol molecules, can associate via $\mathrm{O}-\mathrm{H}$...O bonds in rather elongated, layer-like structures. 


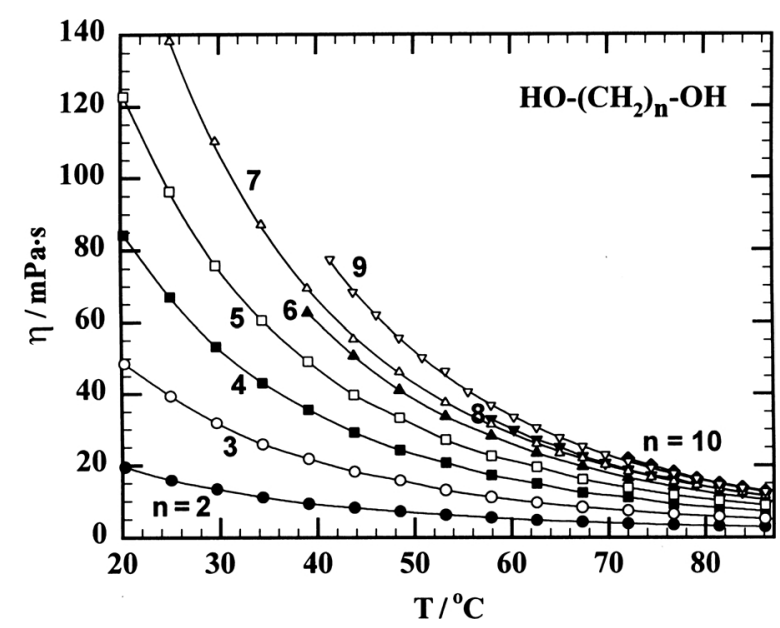

Fig. 2. Temperature dependences of the viscosity of $1, n$ alkanediols. The solid lines represent the best fit of (3) to the experimental viscosity data.

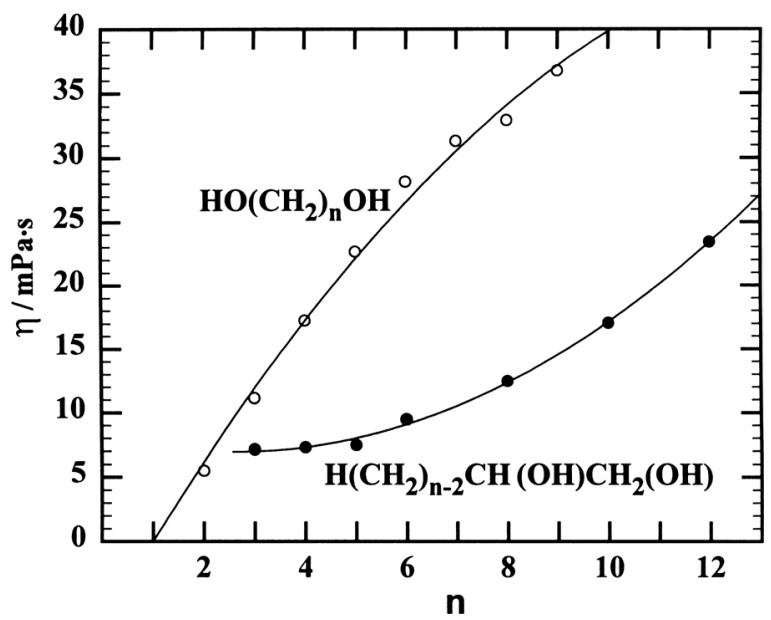

Fig. 3. Viscosity of 1,2- and 1,n-alkanediols as function of the length of the alkane chain, at $58^{\circ} \mathrm{C}$.

Figure 3 shows that the different intermolecular hydrogen bonded structures formed by 1,2- and 1, $n$-alkanediols manifest themselves in quite different viscosity dependences on the length of the alkane chain of the compounds. It is worth noticiting that the extrapolation of the experimental $\eta(n)$ dependence of $1, n$ alkanediols to $n=1$, i.e. to methanediol, $\mathrm{HOCH}_{2} \mathrm{OH}$, results in a very low viscosity. Of course, the data do not allow to estimate the viscosity of methanediol with a high precision, but the fact is puzzling because this diol, when pure, is very unstable, and perhaps our data can be useful in understanding that instability.

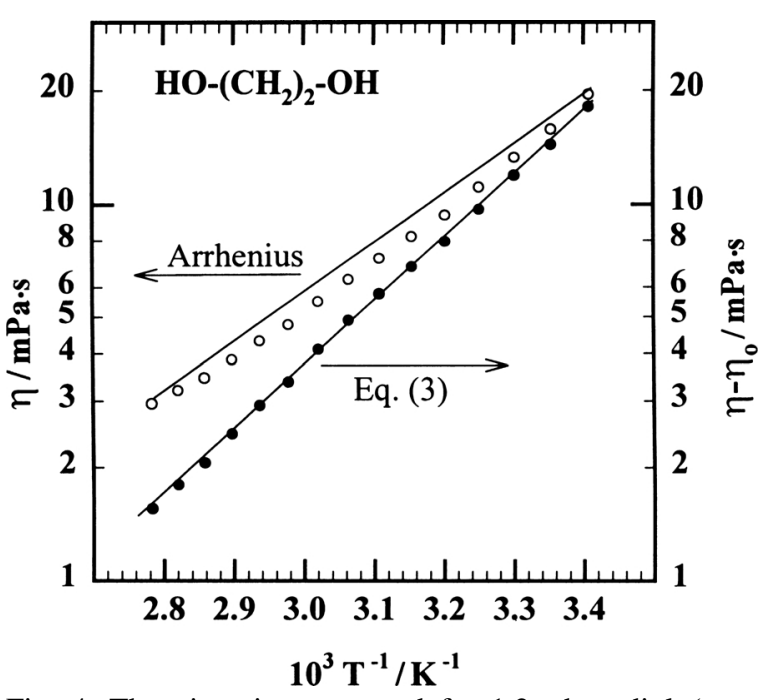

Fig. 4. The viscosity measured for 1,2-ethanediol (open points) as a function of $T^{-1}$ shows non-Arrhenius behavior. Subtraction of the constant value $\eta_{\mathrm{o}} \approx 1.4 \mathrm{mPa} \cdot \mathrm{s}$ makes the results quite good linear, as predicted by (3).

The experimental viscosity data presented in Figs. 1 and 2 do not quite fulfill the Arrhenius dependence

$$
\eta(T)=\eta_{\mathrm{A}} \exp \left(\frac{E_{\mathrm{A}}}{R T}\right)
$$

As an example, the data for 1,2-ethanediol are presented in Figure 4. Two conclusions are in common practice in the literature. Either one ignores the experimental nonlinearity in the $\ln \eta$ vs. $T^{-1}$ dependence, and the activation energy is calculated as the mean slope of the dependence. Or it leads to the introduction of some modifications of the Arrhenius equation. In the 1920's Vogel and Fulcher proposed the equation $[13,14]$

$$
\eta(T)=\eta_{\mathrm{VF}} \exp \left(\frac{D T_{\mathrm{o}}}{T-T_{\mathrm{o}}}\right),
$$

in which three fitting parameters $\left(\eta_{\mathrm{VF}}, D\right.$ and $\left.T_{\mathrm{o}}\right)$ occur. Equation (2) is used with success for the empirical description of the viscosity dependence on temperature for many liquids, specially in the supercooled state $[15,16]$.

Another modification of the Arrhenius equation is based on the following experimental fact observed for many liquids of different structure and interactions: the axis $\eta=0$ (as in the Arrhenius equation) is not the real asymptotic axis for the viscosity measured with increasing temperature. Of course, a strict discussion on 


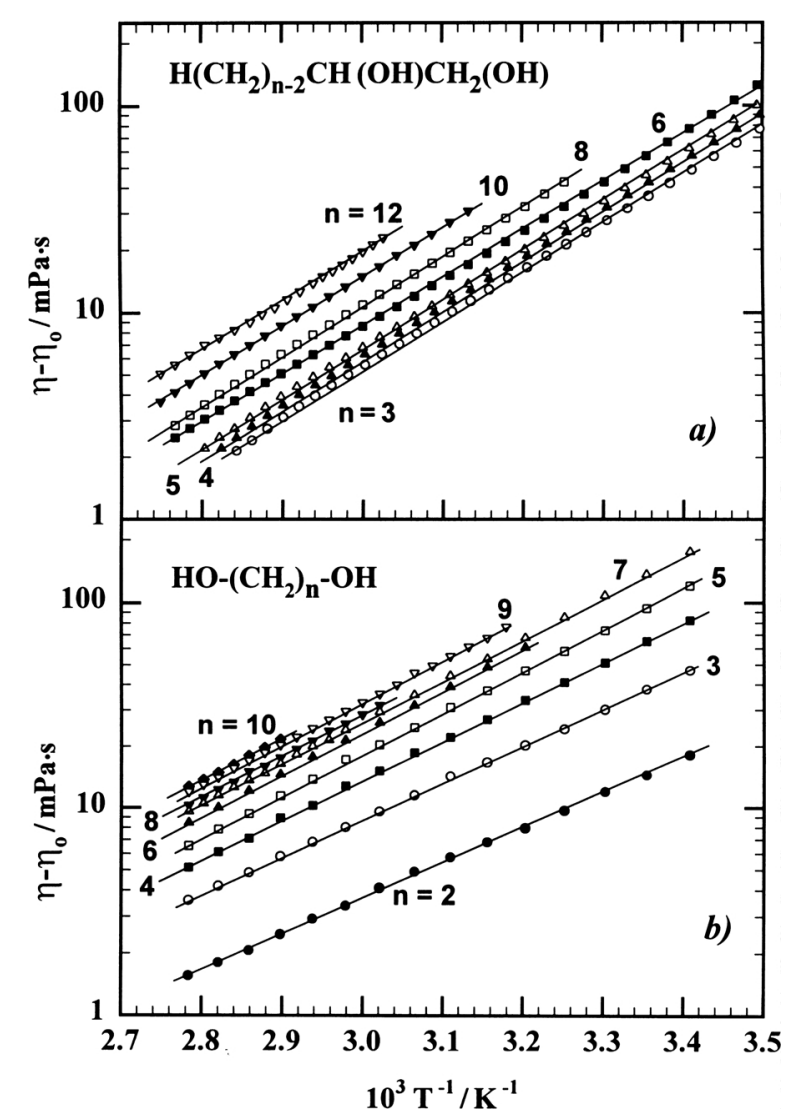

Fig. 5. Arrhenius-like plots for the viscosity of 1,2-(a) and $1, n$-alkanediols $(\boldsymbol{b})$.

that problem is rather difficult because, in practice, the temperature range $\Delta T$ of the viscosity measurements is very narrow if compared to the whole range of possible $T$ variation $\left(T_{0}, \infty\right)$; for a typical liquid $\Delta T$ does not exceed $100 \mathrm{~K}$. Nevertheless, the residual value of the viscosity, of the order of $1 \mathrm{mPa} \cdot \mathrm{s}$ (probably depending on the compound and the experimental method used), seems to be a common feature of different liquids. In such circumstances, the following modification of the Arrhenius equation is possible [17]

$$
\eta(T)=\eta_{\mathrm{A}} \exp \left(\frac{E_{\mathrm{A}}}{R T}\right)+\eta_{\mathrm{o}}
$$

The equation contains three fitting parameters: $\eta_{\mathrm{A}}, E_{\mathrm{A}}$ and $\eta_{\mathrm{o}}$. It was shown in [17] that (2) and (3) reproduce the experimental temperature dependence of the viscosity of different liquids with the comparable reliability. However, (3) is interesting because a very useful conception of the activation energy is conserved here.

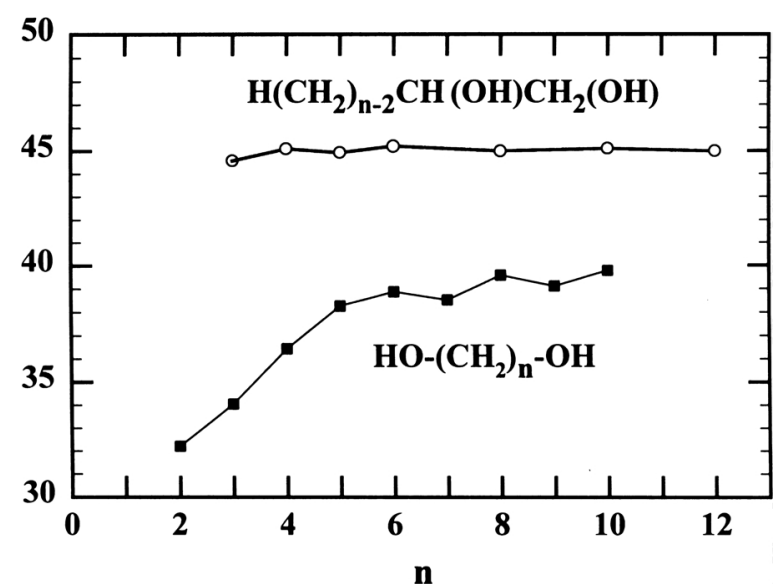

Fig. 6. Viscosity activation energy as a function of length of the alkane chain in 1,2- and $1, n$-alkanediols molecules.

For thermally activated processes, as the viscous flow, the value of the activation energy gives directly the temperature dependence of the process. The problem is quite important as constantly a great effort is undertaken in finding for liquids (such as oils) an appropriate value of the viscosity with a temperature dependence as weak as possible, i.e. with the low activation energy of the viscosity. Besides, quite significant conclusions on the molecular level can be drawn from a comparison of the activation energy of the viscosity with other activation energies, as obtained from dielectric relaxation studies, for example.

The solid lines in Figs. 1 and 2 represent the best fit of (3) to the experimental data.

The essential difference between the Arrhenius approach and that of (3) is presented in Figure 4. If one takes into account the viscosity background, i.e. if one subtracts the constant $\eta_{\mathrm{o}}$ from the experimental viscosity, the dependence $\ln \left(\eta-\eta_{\mathrm{o}}\right)$ on $T^{-1}$ is quite linear and the activation energy can be determined not so optionally as from the Arrhenius $\ln \eta$ on $T^{-1}$ dependence. Figure 5 shows the dependences of $\ln \left(\eta-\eta_{\mathrm{o}}\right)$ on $T^{-1}$ for all diols studied.

The activation energy of the viscosity as a function of length of the alkane chain in 1,2- and 1,n-alkanediol molecules is presented in Figure 6. In case of the 1,2-alkanediols, the activation energy is practically independent of the molecular length. It certainly means that the hydrogen bonded intermolecular entities of the same structure (the micelle-like) are created independently of the alkane tail length in the diol molecule. The results obtained for the $1, n$-alkanediols show that 
the distance between two end $\mathrm{O}-\mathrm{H}$ groups is essential for the liquid structure up to about six $\mathrm{CH}_{2}$ groups in the alkane chain, and for $n>6$ the intermolecular entities of the layer-like structure are dominant. It is inter-

[1] D. J. Evans, Molec. Phys. 34, 103, 161 (1977).

[2] S. Hess and H. J.M. Hanley, Phys. Lett. A, 98, 35 (1983).

[3] H.-M. Koo and S. Hess, Physica A, 145, 361 (1987).

[4] R. Edberg, G. P. Morriss, and D. J. Evans, J. Chem. Phys. 86, 4555 (1987).

[5] C. Hoheisel, J. Chem. Phys. 86, 2328 (1987).

[6] D. Levesque and L. Verlet, Molec. Phys. 61, 143 (1987).

[7] G. Marchal, J.-P Ryckaert, and A. Bellemans, Molec. Phys. 61, 33 (1987).

[8] R. Vogelsang, G. Hoheisel, and M. Lucas, Molec. Phys. 64, 1203 (1988).

[9] S. Sarman, J. Chem. Phys. 103, 10378 (1995). esting to notice that 1,2-ethanediol, which, in principle, belongs to both 1,2- and 1,n-alkanediols, assertively shows the viscous properties corresponding to the homologous series of $1, n$-alkanediols.

[10] W. Allen and R. Rowley, J. Chem. Phys. 106, 10273, (1997).

[11] T. Lech, G. Czechowski, and J. Jadżyn, J. Chem. Eng. Data 46, 725 (2001).

[12] J. Jadżyn, G. Czechowski, and T. Stefaniak, J. Chem. Eng. Data 47, 978 (2002).

[13] H. Vogel, Phys. Z. 22, 645 (1921).

[14] G. S. Fulcher, J. Amer. Ceram. Soc. 8, 339 (1923).

[15] L. R. Cook, H.E. King, C. A. Herbst, and D. R. Herschback, J. Chem. Eng. Phys. 100, 5178 (1994).

[16] R. Bhmer, K.L. Ngai, C. A. Angel, and D. J. Plazek, J. Chem. Eng. Phys. 99, 4201 (1993).

[17] J. Jadżyn, G. Czechowski, and T. Lech, Acta Phys. Polon. 101, 495 (2002). 